\title{
COMMUNICATIONS
}

\section{MONOCULAR DIPLOPIA*}

\author{
BY \\ E. F. FINCHAM \\ Northampton College of Advanced Technology, London
}

SLIGHT doubling of monocular vision can be detected by a large proportion of subjects with good visual acuity and keen perception. The condition has been mentioned in the literature on visual optics but, except in cases in which it is sufficient to reduce vision and may be caused by trauma or progressive changes in the eye media-e.g. early states of cataract, it has attracted little interest. Helmholtz (1866) mentions that the crescent moon appears to many people to have multiple horns, and Emsley (1952) has described a multiple image (monocular polyopia) of a small source of light seen by a myopic eye. Nowadays, the town-dweller is more likely to notice the effect when viewing a distant illuminated sign against a dark background. Usually one image is fainter than the other and hence may frequently be unnoticed.

For this reason visual acuity, as measured by the usual methods, is seldom affected, and most subjects in whom monocular diplopia can be demonstrated under the right conditions have not previously been aware of it. Doubling is not so noticeable with dark objects on a light background, as, for instance, printed type. A point of special interest is that all these cases are similar in that the doubling is almost always approximately in the vertical direction and is homonymous. This is shown by the disappearance of the upper image when the upper part of the pupil is covered. The diplopia occurs as though a very weak prism with base-apex direction vertical was placed across part of the pupil. Frequently only one eye is affected.

\section{Diagnosis}

\section{(a) Subjective Methods}

A convenient laboratory test for the presence of diplopia is to show the patient a bright line on a dark background. This is provided by a slit aperture about $2 \mathrm{~mm}$. in width and $20 \mathrm{~mm}$. long cut in the closed end of a cylindrical vessel, e.g. a preserve can. Behind the slit is fixed a piece of ground glass and inside the vessel is placed a low-power electric lamp. In a darkened room this will show a short line of light which may be varied in

* Received for publication January 18, 1963. 
brightness by altering the distance of the lamp from the aperture or by interposing neutral filters. This test object should be viewed with one eye from distances between 3 and 6 metres. The vessel may be rotated to show the meridian in which the greatest diplopia occurs.

Many subjects will detect the presence of astigmatism by the line of light becoming progressively blurred as one meridian is approached. Apart from this the line may appear distinctly doubled in some positions, a dark space showing between two images. In some cases the effect is more marked if the eye is in effect made myopic by interposing a weak positive lens. Some subjects have reported that diplopia is more apparent if the luminous line is coloured, for instance, green. This may be due to the reduction of the blurring effect of chromatic aberration or again to the introduction of a small amount of relative myopia.

In cases which show diplopia, some simple experiments can be made. Moving a card or stop with its edge perpendicular to the direction of doubling close to the eye, so that parts of the pupil are covered, will show the optical nature of the defect. If a pinhole aperture is placed close to the eye, one image is eliminated, and if the aperture is then moved in the direction of doubling the image will appear to jump. This shows that the defect is not caused by a normal refractive error, i.e. lens power, which would cause a gradual and not a sudden movement of the image. Usually, the image jumps upward as the pinhole is moved up. This observation leads to the conclusion that there is a prismatic difference between two parts of the pupil, either a prism base down in the upper part, or a biprism with the bases joined across the pupil aperture.

The measurement of the amount of doubling cannot be made with great accuracy as the angles to be measured are quite small and success depends so much upon the subject's acute perception. In a few cases in which diplopia is clearly apparent and the secondary image is strong, the subject is able to compare the doubling of a small source of light with the size of a Snellen test letter, e.g. the 6-metre letter subtends an angle of $5 \mathrm{~min}$. at 6 metres. Another fairly effective method is for the subject to view two parallel illuminated slit apertures of which the separation can be varied. The separation and the distance of viewing are adjusted until three bright lines are seen. The doubling of the image is then equal to the angular separation of the illuminated slits. A third method which has been tried with some success is the direct approach of correcting the prismatic effect of part of the pupil. The previous methods had shown that it was rare for the angle of doubling to be greater than $5 \mathrm{~min}$. To correct this by means of a prism placed part way across the pupil presents some difficulties. The power of the prism would be about $0 \cdot 15$ prism dioptres, and its edge would need to be very thin so that it would not occupy much of the pupil space. As an exercise in glass working, this proved to be too difficult. Instead, a lens of $+0 \cdot 12$ dioptre power was worked as thin as possible-not more than $0.5 \mathrm{~mm}$. centre 
thickness-and was cut to a rectangle so that the optical centre was at different distances from the four edges. Thus, by placing different edges of the lens across the pupil, four prismatic effects due to decentration could be produced. In this method the decentration and hence the prismatic effect varies over that part of the pupil affected by the lens, but the optical centre is marked and its displacement from the middle of the part of the pupil concerned can be taken. As an example, placing one edge of the lens about half-way across the pupil brings the optical centre $7 \mathrm{~mm}$. above the centre of the part of the pupil which is covered by the lens. This 7-mm. decentration of a $0 \cdot 12$ dioptre lens causes a prismatic effect of $0.084 \triangle$, which equals approximately $3 \mathrm{~min}$. In the case of one subject this was found to eliminate the diplopia; in other cases more decentration of the lens was required. It is obvious that only a few cases could be corrected in this way with the single lens which was available.

\section{(b) Objective Methods}

Retinoscopy.-The influence of irregular refraction on the pupil illumination in retinoscopy depends on the type of retinoscope illumination. The plane mirror retinoscope reflecting light from a fairly large source, or a luminous instrument giving the same type of broad illumination of the retina, will not show small irregularities of refraction. To make these apparent a luminous retinoscope which produces on the subject's retina a very small luminous spot or line, as does the streak retinoscope, must be used. Then, as the point of reversal is reached, i.e. the subject's retina is made conjugate with the observer's pupil by means of lenses, the bright glow in the subject's pupil is sometimes seen to be broken by dark areas. This is the result of irregular refraction.

Most cases of monocular diplopia show this split reflex in retinoscopy. In the typical case an approximately horizontal dark band appears across the pupil. When the light from the retinoscope is made to move in a vertical direction, the light in the upper part of the pupil moves against, and that in the lower part moves with, this direction. The usual interpretation of this appearance is that the upper part of the pupil is relatively myopic and the lower part hypermetropic. Generally the effect is seen only within a small dioptric variation, a $+0.5 \mathrm{D}$ lens before the eye often giving a uniform "against" movement, and $-0.5 \mathrm{D}$ lens a uniform "with" movement. Nevertheless, this irregularity is seen to occur in many cases and constitutes one of the difficulties of retinoscopy, preventing an exact estimation of the refractive error from being made by this method.

The Coincidence Optometer (Fincham, 1937).- This instrument gives an objective measurement of the refraction of the eye, but because of the principle on which it is based it scans the pupil area, measuring it piecemeal. 
For this reason it is useful in the study of irregular refraction, and many such cases were found soon after the optometer was developed. What had not been noticed before was that, in a number of cases in which the principal meridians were horizontal and vertical, a difference in power existed between the upper and lower parts of the pupil. Thus, in these cases, two possible prescriptions could be written from the optometer readings. In general, the refraction in the horizontal meridian was fairly uniform, so that the same spherical correction could apply in both prescriptions, but the cylindrical element was different in the upper and lower parts. In practice it was almost always found that the prescription with the weaker cylinder agreed with the best correction found by subjective test. So much was this the rule, that the author's routine practice in these cases was to try the weaker correction first, and it was seldom necessary to modify it.

This condition has been found in the cases of monocular diplopia which have been studied recently. Of seventy eyes that have been measured with the optometer in this investigation, 33 were found to have a significant difference of refraction between upper and lower halves of the pupil. Differences of optometer reading of less than $0.5 \mathrm{D}$ were not thought to be significant and in these diplopia was not detected. In thirty eyes the measurement for the upper part of the pupil was more myopic than the lower part by $0.5 \mathrm{D}$ or more, 37 eyes showed only a slight difference although many were astigmatic.

In those cases in which the excess of refraction in the upper part of the pupil was significant, the difference was as follows: 1.25 to $1.75 \mathrm{D}$ in four eyes, $1 \mathrm{D}$ in eight eyes, $0.75 \mathrm{D}$ in eight eyes, and 0.5 D in ten eyes.

The opposite condition, i.e. top of pupil less myopic than bottom, was found in only two eyes (different subjects), and the difference in these cases was $0.5 \mathrm{D}$. In three eyes, the principal meridians were oblique, i.e. $45^{\circ}$ and $135^{\circ}$; in two of these there was no irregularity and in the other the lower $135^{\circ}$ region of the pupil was more myopic by $1 \mathrm{D}$ than the upper part. The subject was conscious of diplopia with this eye, the maximum doubling being at $135^{\circ}$.

\section{Site of the Irregularity}

The results of the simple experiments that have been described here leave no doubt that this diplopia is caused by an anomaly of the optical system of the eye. The effect could be due to the anterior surface of the cornea or to either surface of the crystalline lens. To cause the effects which are seen when a pinhole is moved before the eye, it would be necessary for one of the surfaces to be divided by a horizontal edge with one part of the surface tilted vertically with respect to the other. There are two direct methods of testing the corneal surface for such a defect. The first of these is objective, by observation of reflections by means of a keratometer or photokeratograph. Photographic records of the image reflected by the cornea have been made on a number of subjects with diplopia, but in no case was an irregularity of the 
surface found which would account for the defect. The other method of testing the corneal surface is by fitting a contact lens, but when this was tried on one subject it was found that diplopia persisted when the lens was worn. Therefore, irregularities of this surface were not the cause of the diplopia.

The lens surfaces can also be studied by observation of reflections. The third Purkinje image, reflected from the front surface of the lens, is rather diffuse, but with suitable illumination it is reasonably clear and might be expected to show a defect of the amount we are seeking. Doubling of this image from certain small parts of the surface has been observed in some subjects in the past. This doubling occurs at very limited areas. As the angle of reflection is changed, the image is seen suddenly to split and then to jump to a different position as though it was passing a ridge or trough on the surface. The effect is thought to be due to the suture structure of the lens. This doubling is not to be confused with the multiple images caused by reflection at the surfaces of discontinuity within the lens described by Hess (1911) which are seen more particularly in older eyes. In such cases the images become more separated as the angle of reflection is increased. The author has never seen doubling of the images from the posterior surface of the lens. In the present investigation one eye was examined by this method. The subject experienced distinct diplopia and showed a difference of refraction between the upper and lower parts of the pupil. It was this subject who was tested with a contact lens. No doubling of the image from either the front or back surface of the lens could be detected, although the eye was turned to bring the images to various parts of the pupil.

\section{Discussion}

To interpret the findings by the optometer, it is necessary to consider the principle of the instrument. A narrow beam of light from a target upon the axis of the eye is made to enter the pupil at a decentred position. The refraction of the eye is measured by adjusting the angle at which this beam of light must enter the pupil, so that a retinal image of the target also lies upon the axis. The entrance pupil has a fixed decentration from the axis and the adjustment is made by moving the target along the axis relative to a lens which is before the eye. When the target is at the principal focus of this lens, the narrow beam of light will enter the eye parallel to the axis, and thus if the eye is emmetropic the retinal image will also be on the central axis. In myopia such a beam will cross the axis before it reaches the retina, so that the image will be decentred, while in hypermetropia the light will not reach the axis and the image will be displaced to the other side. To bring the image to the central position in ametropia the target must be moved along the axis so that the beam of light must reach the eye at an angle with the axis, divergent in myopia and convergent in hypermetropia. The viewing system of the apparatus contains a sensitive optical device for determining when the retinal image is central. 
It will be seen that the displacement of the retinal image, which is the criterion in this method of measurement, may be brought about not only by variations in lens power in the part of the eye which is being tested, but also by prismatic effect. If in the upper part of the pupil there is prismatic power with the base of the prism down, the retinal image would be displaced downwards when the light is entering through this part of the pupil. It could be brought on to the axis by a forward movement of the target in the instrument (to make the beam of light entering the eye diverge from the axis). This adjustment would be read as showing a condition of myopia.

The adjustment of the optometer required to give a reading of $-1 \mathrm{D}$ causes the light entering the eye to diverge from the axis by approximately $4.5 \mathrm{~min}$. Therefore we should expect subjects in whom the upper part of the pupil appeared to be more myopic than the lower part by $1 \mathrm{D}$, to have diplopia with the two images separated by about $4.5 \mathrm{~min}$. As we have said, the subjective measurement of the angle of doubling was somewhat uncertain so that very close correspondence with the prediction by the optometer could not be expected. Usually the perceived angle was the greater. For example, the greatest diplopia that was recorded was $10 \mathrm{~min}$; this eye also showed the greatest difference in optometer reading, but this was $1.7 \mathrm{D}$, which we would expect to give diplopia of $7.5 \mathrm{~min}$. The best agreement was that for an eye showing diplopia of $5.9 \mathrm{~min}$. which was found by the optometer to have a prismatic difference in the two parts of the pupil of $5.5 \mathrm{~min}$.

Regarding the site of the optical defect which causes diplopia, the absence of irregularities of the image reflected from the anterior surface of the cornea and the failure of a contact lens to correct the effect lead to the conclusion that this surface can be eliminated from the possible causes. The posterior corneal surface has relatively small optical power and its deformation sufficient to cause diplopia is unlikely. Considering the structure of the crystalline lens, we are led to suspect this part of the optical system as being responsible for the defect. The failure to detect doubling of the catoptric images from the lens surfaces of one eye, although in a definite case of moderate diplopia, cannot be taken as conclusive evidence that irregularities in these surfaces are not sometimes the cause of diplopia. It is possible that the image from the posterior surface is too small to show minute amounts of doubling by the method of examination which was used.

The other possible cause is an irregularity in the lens substance. It is well known that the radial sutures of the lens are responsible for the star figure which small sources of light appear to assume to almost all subjects. The shape of the star is rarely symmetrical, frequently one ray is longer than the others, and the star varies in form from one eye to another. There are probably small local differences in refractive index between the lens fibres and the cement substance which cause some light to be scattered. On the other hand, diplopia is the result of a definite prismatic effect. If this is not 
caused by a relative tilting of part of one or more of the optical surfaces, it must be due to a difference in refractive index occurring along a line which divides the pupil into two parts.

The relatively high incidence of this condition in otherwise normal eyes of young to middle-aged subjects is an unexpected finding. An effect such as would be caused by a vertical prism with its base down across the upper part of the pupil was found in 43 per cent. of the seventy eyes which were tested. It seems, therefore, that there is a strong tendency for the lens structure to develop in this way. No attempt has been made to find the position of the division of the prism element, i.e. the base of the biprism, relative to the pupil centre. The upper image is generally the weaker one, in fact often only a feeble ghost, so we may assume the division is above the centre of the pupil.

Although the effects which have been described are small and are seldom noticed in the clinical measurement of visual acuity, they must affect the finer perception of detail. This study was the result of an inquiry by a microscopist, who was trying to resolve very fine detail and noticed that bright images were slightly doubled in the vertical direction. The existence of a faint secondary image above the main image appears to offer an explanation of an effect which the author noticed when using the Landolt's ring test many years ago. In a project to study irregular astigmatism it was decided to use this test, and a single ring of the usual design was mounted so that it could be rotated to bring the gap into any meridian. With the ring in any set position it was slowly brought forward towards the subject until he could state correctly the position of the gap against a protractor. It was found that many subjects could give the correct answer when the test object was at a greater distance if the gap was approximately vertical upwards than if it were downwards in the same meridian. This finding is not explained by the usual theory of resolution or the effect of refractive errors.

In this test it is not necessary for the subject actually to resolve the gap in the ring; he need only recognize that a part of the black circle is less intense than the rest. How the ability to do this can be helped by a ghost image displaced in the direction of the gap can be shown by a simple experiment. A lantern slide of two Landolt rings, one with the gap at the top and the other at the bottom, is projected to give an image about the size of a 6-metre test letter. A weak prism, base-apex line vertical, is then placed with its base upwards over a small part of the projection lens. The effect is to produce a weak ghost image above the main image (see Figure, overleaf). The power of the prism should be such as to cause a displacement rather less than the diameter of the ring. If now this image is observed from beyond 6 metres and slowly approached, the ring with the gap in the direction of the doubling will be recognized first. The Figure shows why this happens.

The main image of the ring is reduced in intensity by the overlapping of the white background, except where the secondary image of the ring and the main 

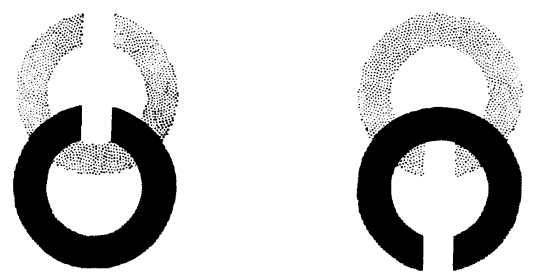

FIGURE.-Effect of diplopia on appearance of Landolt ring. For description see text.

image coincide. Thus two dark areas appear; when these are adjacent to the gap in the ring they help in its recognition, but when they are on the other side they are of no advantage or may even cause confusion. It will be seen from the Figure that the effect which is described occurs when the angle of diplopia is about $4 \mathrm{~min}$. This is a common condition.

\section{Summary}

Suitable tests have shown that slight monocular diplopia occurs in a large proportion of eyes which have normal visual acuity. It has been found in over 40 per cent. of seventy eyes of subjects between the ages of 18 and 45 . Generally one image is fainter than the other and is almost always displaced approximately vertically upward. The usual amount of displacement is between 3 and $6 \mathrm{~min}$. of arc. Smaller amounts are probably undetected. Subjective tests and objective measurement show that in such cases the optical system of the eye has a prism of power about $0 \cdot 1^{\Delta}$ in the upper part of the pupil. The prism base is down and the base-apex direction approximately vertical. This prism apparently covers only a small part of the pupil and so causes a faint secondary image. As irregularity cannot be detected in the corneal or lens surfaces, it is thought that the effect is due to a refractive index difference in the lens substance. One effect of this irregularity is to cause asymmetry in the resolving power of the eye, e.g. a Landolt ring is more easily recognized when the gap is upward, i.e. in the direction of the ghost image.

The author's thanks are due to Mr. P. Fairbanks for making the very thin lenses used in the measurement of the prismatic power.

\section{REFERENCES}

Emsley, H. H. (1952). “Visual Optics", 5th ed., vol. 1, p. 414. Hatton Press, London.

Fincham, E. F. (1937). Proc. phys. Soc. (Lond.), 49, 456.

HelmhOlTZ, H. vON (1866). "Handbuch der physiologischen Optik". "Helmholtz's Treatise on Physiological Optics", trans. from 3rd German ed., ed. J. P. C. Southall (1924), vol. 1, p. 191. Optical Society of America.

Hess, C. (1911). "Pathologie und Therapie des Linsensystems", in "Graefe-Samisch Handbuch der gesamten Augenheilkunde", 3rd. ed., teil 2, kap. 9. Engelmann, Leipzig. 